\section{TREATMENT OF OTOSCLEROSIS BY STAPEDECTOMY}

BY

\author{
A. W. MORRISON, M.B., Ch.B., F.R.C.S., D.L.O. \\ Consultant E.N.T. Surgeon, Whipps Cross Hospital, \\ London
}

[With Special Plate]

The surgical treatment of otosclerosis has changed since the open one-stage fenestration was introduced by Lempert (1938). The principal disadvantages of the operation were the creation of a mastoid cavity resulting in chronic otorrhoea in $13 \%$ of cases (Hall, 1958), occasionally troublesome post-operative vertigo, and closure of the fenestra in the lateral semicircular canal within the first few months of operation. The nonphysiological nature of the operation precluded all but a few normal hearing results. Hilleman and Shambaugh (1959) have analysed 10-17-year results in a large series and found $43 \%$ with hearing maintained about the 30-decibel level.

Indirect mobilization of the fixed stapes as described by Rosen $(1953,1954)$ was the next advance. If it was successful the patient had an intact functioning ossicular chain and near normal hearing. The operation had few complications, but regrettably few successes. In my own experience of some 100 operations of this type, dramatic improvement in hearing occurred in only $25 \%$. Published results vary considerably, but Scheer (1957) with $33 \%$ of good results is representative. Recently, however, Bellucci (1961) has examined five-year results and found only $12 \%$ of patients with serviceable hearing.

Arising from this disappointment many modifications of Rosen's operation have been described, such as anterior crurotomy (Fowler, 1956), fenestration of the oval window (Rosen, 1957), partial stapedectomy and prosthetic reconstruction (Juers, 1959; Hough, 1960 ; Bellucci, 1961), and many others. Though results have been better, the major problem remains-re-ankylosis of the stapes footplate. Portmann and Claverie (1959), from Bordeaux, described their "interposition" operation, removing the footplate, vein-grafting the oval window, but still utilizing part of the stapes, with greatly improved results.

The more radical total stapedectomy followed by reconstruction of the ossicular chain with a polythene prosthesis was pioneered by Shea $(1958,1959,1960)$.

The purpose of the present paper is to outline the operative procedure and analyse the early results of this Shea-type operation. The numbers are admittedly not large, and the time interval of up to 18 months is short in terms of permanent cure ; but it is felt that the results are sufficiently encouraging to warrant publication of the experience.

\section{Operative Procedure}

Stapedectomy is a permeatal operation carried out with the aid of a suitable binocular operating microscope.

The middle ear is entered postero-superiorly after raising a skin flap from the posterior meatal wall to the tympanic annulus. The chorda tympani is divided if it obscures vision, and enough bone is removed to give access to the long process of the incus, the stapedius tendon, and the stapes. The round window is inspected. (Special Plate, Fig. 1).

The incudo-stapedial joint is divided and the stapedius tendon cut. The stapes is then dislocated inferiorly on to the promontory and removed. Both crura normally fracture near the footplate-only once has the stapes come away in toto. Before the footplate is removed haemostasis is secured.

The appearance of the footplate and its ease of removal vary greatly, and there is apparently no constant relationship between the degree of deafness and the thickness and ankylosis of the footplate. If thin it is fractured across its centre and first the anterior and then the posterior halves are removed. If moderately thick it is often possible to find an opening into the vestibule at its inferior margin and lever the plate out of the fenestra ovalis. Sometimes the oval-window recess is obliterated by otosclerotic bone, when a micro-drill must be employed to make the fenestra. In this event the superior relationship of the facial nerve and the anteroinferior proximity of the first coil of the cochlea must be remembered. Suction of perilymph is avoided.

To prevent perilymph from escaping and to form a new membrane in the oval window a free vein graft is inserted. An assistant removes a 2-cm. length of vein from the dorsum of the hand or foot, opening the lumen and stretching and drying it during the initial stages of the operation. This simplifies the prompt and accurate positioning of the graft, which otherwise is difficult to handle. Unlike Shea and others I always place the less traumatic vein intima facing the vestibule (Special Plate, Fig. 2).

The operation is completed by placing the new stapes, the polythene prosthesis, in position. Polythene tubing of $1 \mathrm{~mm}$. external diameter and $0.5 \mathrm{~mm}$. bore is usually employed, the length varying from case to case but normally about $0.5 \mathrm{~cm}$. One end of the tube invaginates the vein into the oval window, and into the other end is inserted the lentiform process of the incus, thus anchoring the prosthesis at both ends (Special Plate, Fig. 3).

Finally the skin flap is replaced and the meatus packed with ribbon gauze impregnated with flavine emulsion. The operation is carried out under antibiotic "cover" and the pack removed in five days.

\section{Anaesthetic Technique}

A hypotensive anaesthetic technique is employed to produce as dry an operating field as possible, thus reducing the use of suction, minimizing labyrinthine trauma, and lowering the incidence of post-operative vertigo, vomiting, and cochlear damage. As is known, any hypotensive technique carries a minimal additional risk to the patient, but this has to be balanced against the increased ease of operation and the lowered morbidity from post-operative complications.

In this series the patients were operated on flat, and the hypotensive agent used was hexamethonium iodide, dosage being adjusted according to age, the aim being to produce a systolic blood-pressure between 60 and $70 \mathrm{~mm}$. Hg. Before intubation the larynx and trachea were sprayed with $4 \mathrm{ml}$. of $4 \%$ lignocaine to prevent straining and a rise in venous pressure. After induction, maintenance of anaesthesia was by $3 / 4$ nitrous oxide/ oxygen plus $0.5-1 \%$ halothane. A Gordh needle was kept in a vein during and after operation so that vaso- 
pressors could be given immediately should there be an unexpected fall in blood-pressure. This occurred only twice.

In this series the average operating time was 30 minutes, and there were no complications of induced hypotension. The majority of patients made an uneventful recovery, being discharged on the sixth or seventh day. There was minimal vertigo lasting 24-48 hours, and this was usually positional in origin, but absent at rest.

\section{Selection of Patients}

All the patients had conductive deafness due to clinical otosclerosis, with the pure-tone air-conduction audiogram showing more than a 40 -decibel loss. In $12 \%$ the loss was approximately 40 decibels and in $7 \% 80$ decibels, and in $81 \%$ the loss lay between 50 and 70 decibels. All had a bone-conduction level at least 20 decibels better. The audiometer used was an " amplivox" Model 82 (British Standard). All patients who underwent stapedectomy more than six months ago were included, the total number being 45 .

\section{Results}

It would be impracticable to publish serial audiograms of all patients. An excellent operative result was considered to have been achieved only if the patient's deafness was clinically cured for normal conversation, hearing-aids had been discarded, the air-bone gap had been closed, and the improvement was maintained. In audiometric terms this meant an air-conduction level between 0 and 30 decibels. There were 36 patients $(80 \%)$ in this category. Two patients $(4.5 \%)$ were classed as moderately improved though still clinically deaf. The remaining seven cases $(15.5 \%)$ were failures. These results are summarized in Table $I$.

\begin{tabular}{|c|c|c|c|c|}
\hline & & \multicolumn{2}{|c|}{ Time-interval Since Oneration } & \multirow[b]{2}{*}{ Total } \\
\hline & & $\begin{array}{l}1 \text { Year to } \\
18 \text { Months }\end{array}$ & $\begin{array}{c}6-9 \\
\text { Months }\end{array}$ & \\
\hline $\begin{array}{l}\text { Excellent result } \\
\text { Moderately improved } \\
\text { Unchanged } \\
\text { Worse } \quad . .\end{array}$ & $\begin{array}{l}\cdots \\
\cdots \\
\cdots\end{array}$ & $\begin{array}{r}22 \\
1 \\
1 \\
2\end{array}$ & $\begin{array}{r}14 \\
1 \\
4 \\
-\end{array}$ & $\begin{array}{c}36(80 \%) \\
2(4 \cdot 5 \%) \\
5(11 \%) \\
2(4 \cdot 5 \%)\end{array}$ \\
\hline Totals & . & 26 & 19 & $45(100 \%)$ \\
\hline
\end{tabular}

All the failures manifested themselves within the first three months; and by comparison all patients with an excellent result at three months have so far maintained their improvement, many continuing to show a slight gain in hearing up to six months, especially in the upper frequencies, where there is sometimes an initial postoperative fall.

Audiogram 1 shows the mean collective curve from the 36 excellent results. It will be seen that the average improvement is in the 35-decibel range and that the air-bone gap has been closed. In the majority of these patients there was a striking similarity between the preoperative bone conduction and the post-operative air conduction, and this is now used for estimating prognosis. Audiograms $2-4$ are shown as samples of this. In successful cases tinnitus was lost or greatly diminished.

Of the moderately improved cases, one had a rise from 80 to 50 decibels, while the other improved from 70 to 40 decibels (Audiogram 4). In both cases the improvement was limited by the pre-operative cochlear function.
All of the seven failures had an initial increase in hearing, but had deteriorated before three months. In five of the seven hearing has returned to its pre-operative level, and in two the hearing is worse. The causes of failure are summarized in Table II.

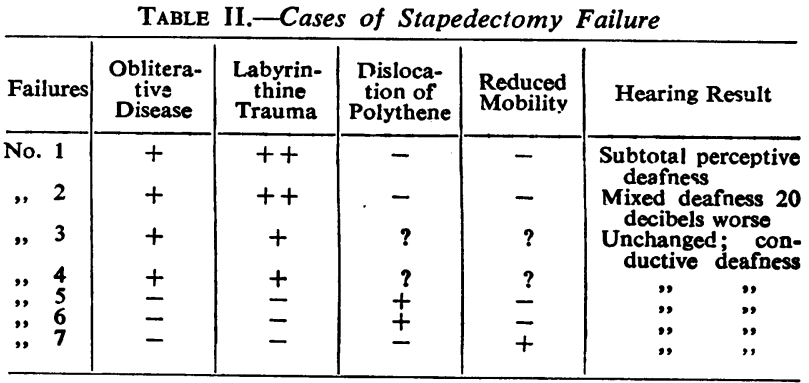

\section{Discussion on Failures}

An excellent hearing result depends upon the avoidance of vestibular and cochlear damage, upon a good articulation between the lentiform process of the incus and the prosthesis, and upon the free mobility of the new ossicular chain. The round window must also be patent-it has twice been found to be partially involved.

In failures Nos. 1, 2, 3, and 4 disease was advanced with dense bone filling the oval-window niche. This necessitated drilling, gouging, and saction with inevitable labyrinthine trauma. These patients all had postoperative vertigo lasting up to two weeks, continuous for a few days and intermittent and positional after this. Operative labyrinthine trauma in one of these four resulted in the only case of subtotal deafness. Yet the finding of this advanced disease is not a contraindication to proceeding with the operation, for two of the excellent results were in similar cases.

Failure No. 3 had post-operative acute suppurative otitis media, which probably contributed to the middleear changes.

In failures Nos. 5 and 6 the cause was disarticulation of the incus-polythene joint, confirmed by re-exploration three months later. These cases were instructive. Both demonstrated absence of inflammatory reaction to the foreign body: the polythene tubes had acquired a covering of thin healthy mucosa with visible bloodvessels running up from the promontory. This confirms the experimental findings in cats by Withers et al. (1961), who also reported that the vein became covered by mucosa laterally and endosteum medially.

Failure No. 7 was due to reduced mobility. As the deafness remained conductive, re-exploration was carried out. The tube was in perfect position and covered by mucosa, but movement at the oval window was almost absent. Failures No. 3 and 4 are awaiting re-exploration. Withers et al. (1961) report a case of active otosclerosis where the vein graft was invaded by osteogenesis some months after operation. This phenomenon, or fibrosis at the oval window, is the likely cause of "reduced mobility" failure.

The exact size and shape of the polythene varies from case to case and may have to be altered to ensure good articulation and mobility. The three modifications required so far have been trimming of its medial end to fit a smaller oval-window recess, expanding its lateral end to enable the lentiform process to articulate, and the making of a right-angled prosthesis for direct 
articulation with the long process of the incus when the lentiform process has fractured. This latter modification has been necessary twice.

\section{General Discussion}

This series, with a satisfactory outcome in $84.5 \%$ of cases, is roughly in line with those of Shea (1959), with $75 \%$ improved, and of Scheer (1961), who had $91 \%$ good results with a similar operation; also with that of Portmann (1961), who had $92 \%$ successes with his transposition operation. Though standards and techniques varied, all these authors employed the same principle-namely, complete removal of the footplate in order to minimize the risk of re-ankylosis. Similarly, most are agreed that the commonest causes of failure are surgical trauma to the labyrinth associated with advanced disease, slipping of the prosthesis, adhesions, and haemorrhage. Haemotympanum has been encountered twice in this series without affecting the final result.

The obvious disadvantage of this type of stapes surgery is the risk of total perceptive deafness. A recent American survey of the subject by Kaplan and Sham-
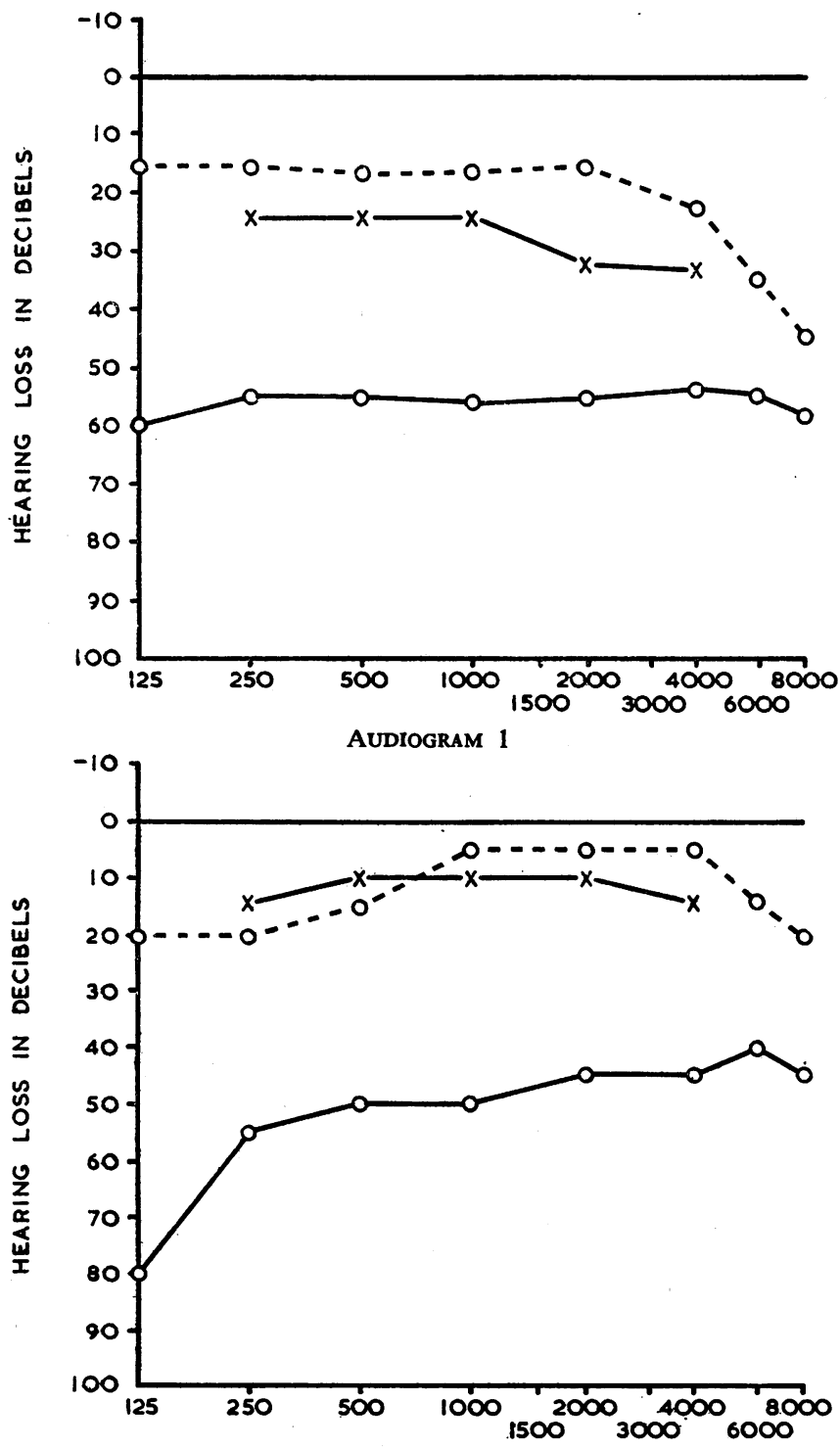

Audrogram 3 baugh (1961) suggests that the incidence of cochlear loss is as high as $4 \%$, compared with about $1 \%$ following fenestration of the lateral canal. Despite this the trend in America is towards the more radical stapedectomy. Portmann (1961) had $2.5 \%$ of total deafness, and one out of 45 in the present series is $2.2 \%$. This figure should diminish with increasing experience.

It is early to judge long-term results, but the high percentage of very satisfactory operations after a year or two is encouraging, and the fact that the prosthesis and vein graft are incorporated into the middle ear is promising.

\section{Summary}

The progress of the surgery of otosclerosis is outlined.

The operation of stapedectomy with vein graft to the oval window and reconstruction of the ossicular chain by a polythene prosthesis is briefly described.

The results in 45 patients, all with conductive deafness due to otosclerosis and with at least a 40-decibel loss on the pure-tone audiogram, followed up for 6 to 18 months, show $80 \%$ with deafness cured and only

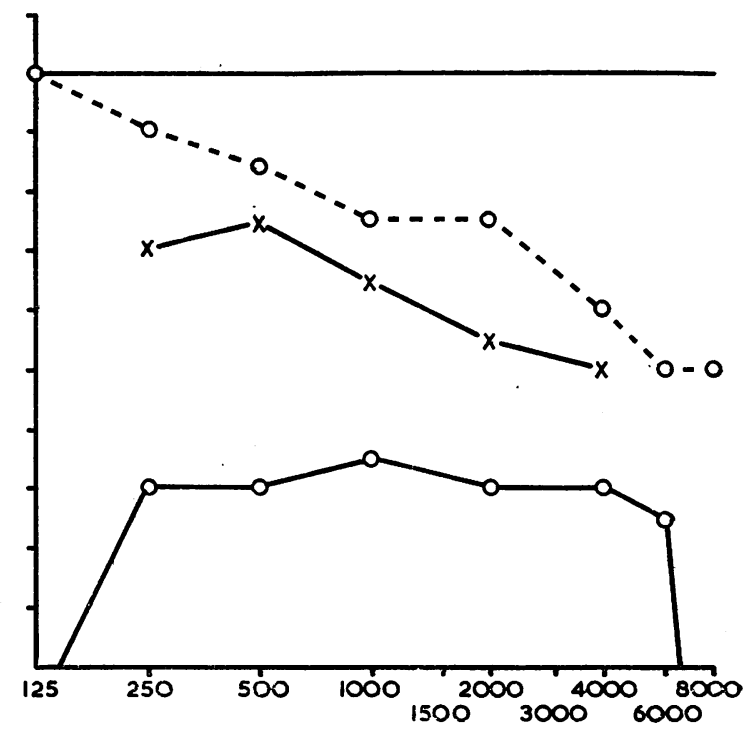

AUdiogram 2

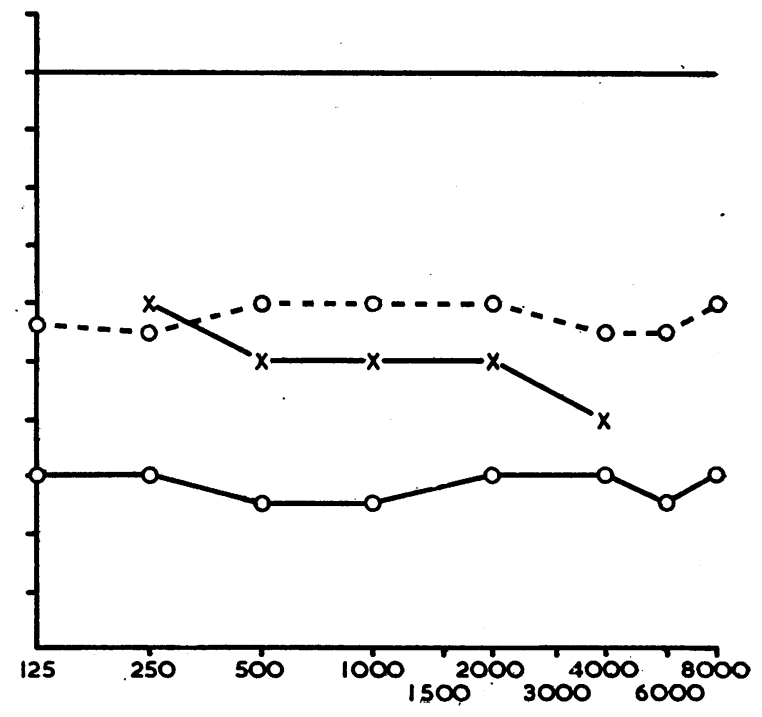

Audiogram 4 
J. FIELDING: SARCOMA INDUCTION BY IRON-CARBOHYDRATE COMPLEXES

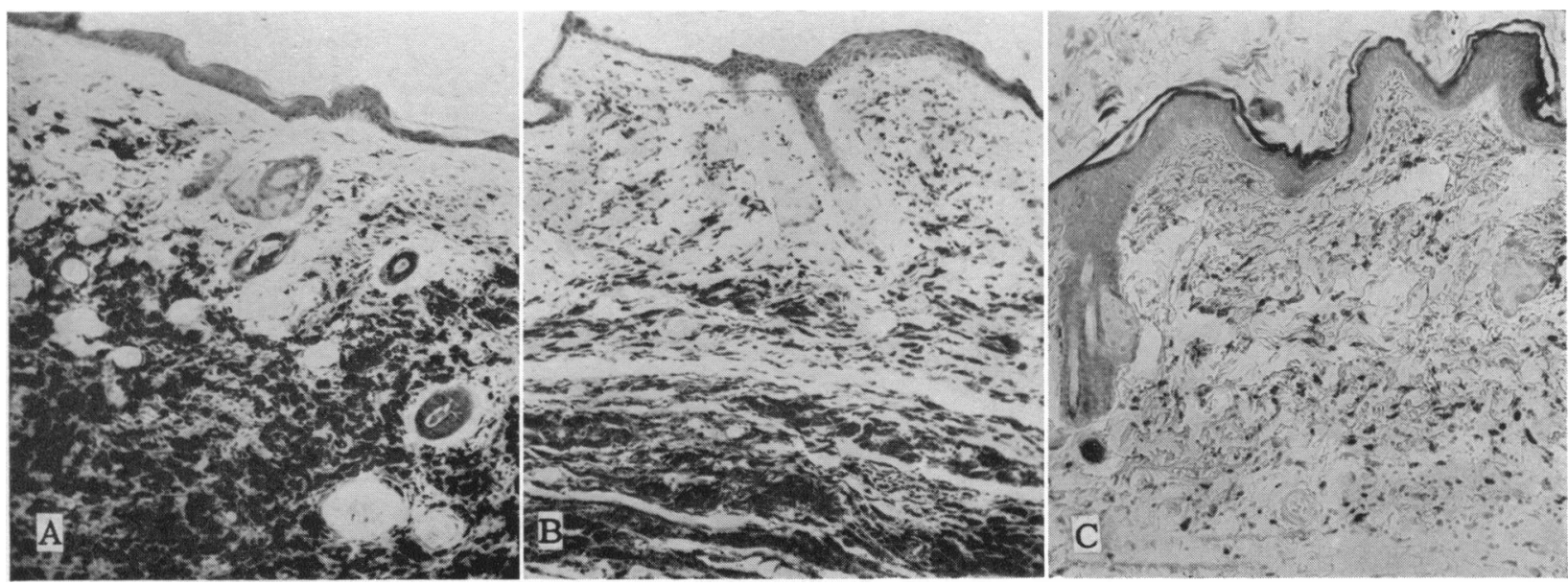

Fig. 1.-Skin and subcutaneous tissue at injection site stained for iron 10 months after injection of (A) 30 mg. iron-dextrin; (B) $28 \mathrm{mg}$. iron-dextran; and (C) $30 \mathrm{mg}$. iron-sorbitol. (Perl's stain. $\times 80$.)

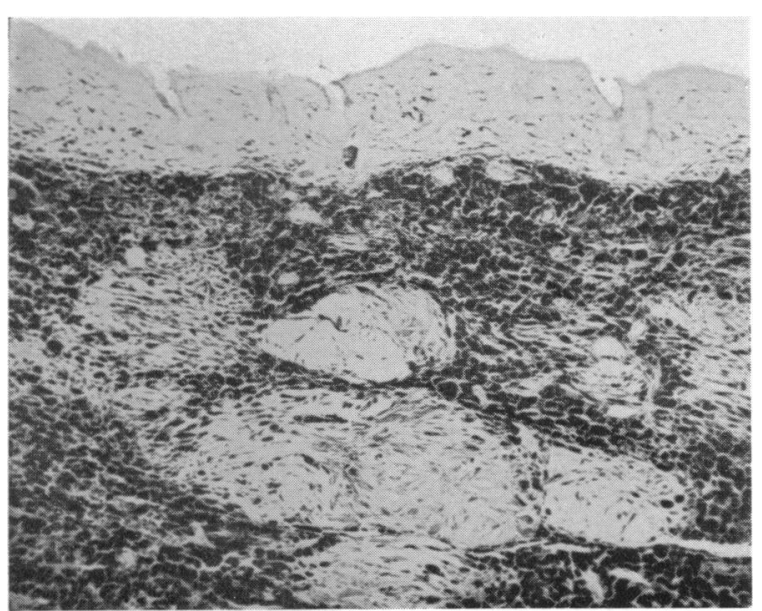

Fig. 2.-Subcutaneous focal fibroblastic proliferation among iron-laden histiocytes 10 months after injection of $30 \mathrm{mg}$. iron-dextrin. (Perl's stain. $\times 48$.)

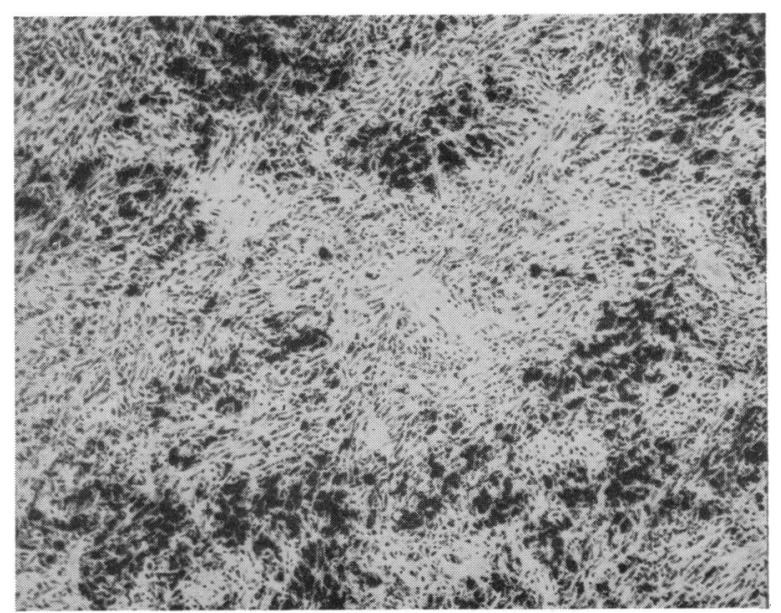

Fig. 3.-Tumour after three weeks' obvious growth, showing islands of iron-containing histiocytes in spindle-cell sarcoma.

(Perl's stain. $\times 80$.)
FIG. 1.-Middle ear opened, showing chorda tympani, long process of incus, stapes, and stapedius tendon.

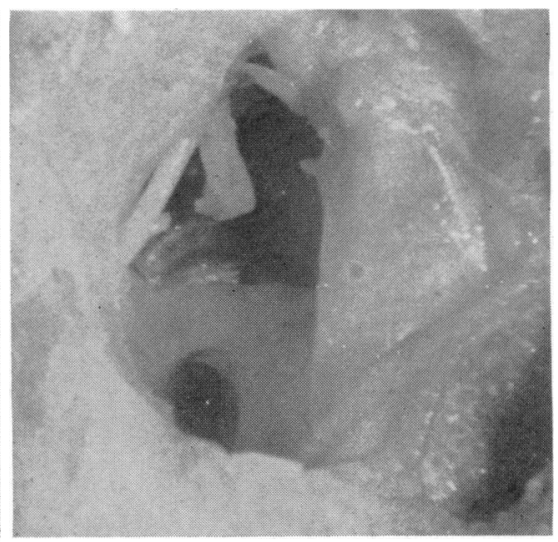

FIG. 2.-Stapes removed. Vein graft covering oval window. Round window clearly seen.

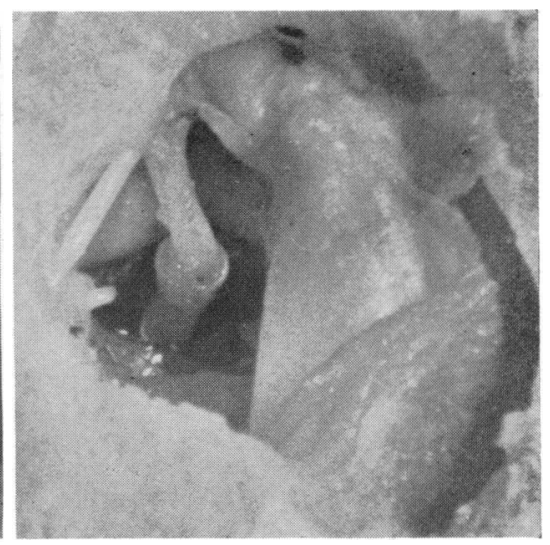

FIG. 3.-Polythene prosthesis in position, articulated with incus. 
$2.2 \%$ with post-operative total deafness. These results are comparable with the few published so far.

Though it is early to assess permanent cure, the indications are encouraging, and it is considered that stapedectomy is at present the operation of choice for suitable cases of otosclerosis.

I wish to express my thanks to Dr. W. K. Slack for his comments on the anaesthetic technique and to the staff of the anaesthetic department of Whipps Cross Hospital for their co-operation. I am also indebted to Dr. D. J. Durcan for his assistance and support throughout the series.

\section{REFERENCES}

Bellucci, R. J. (1961). Arch. Otolaryng., 73, 513

Fowler, E. P., jun. (1956). Acta oto-laryng. (Stockh.), 46, 319.
Hall, I. S. (1958). Proc, roy, Soc. Med., 51, 57

Hilleman, G. A., and Shambaugh, G. E., jun. (1959). A.M.A. Arch. Otolaryng., 69, 136.

Hough, J. V. D. (1960). Ann. Otol. (St. Louis), 69, 571.

Juers, A. L. (1959). Laryngoscope (St. Louis), 69, 1180.

Kaplan, J., and Shambaugh, G. E., jun. (1961). Arch. Otolaryng. 74,522 .

Lempert, J. (1938). Ibid., 28, 42.

Portmann, M. (1961). lbid., 74, 11.

- and Claverie, G. (1959). Otolaryng. pol., 31, 421

Rosen, S. (1953). N.Y. St. J. Med., 53, 2650.

- (1954). Acta oto-laryng. (Stockh.), 47, 78.

(1957). A.M.A. Arch. Otolaryng., 65, 217.

Scheer, A. A. (1957). Ibid., 65, 245.

(1961). Arch. Otolaryng., 74, 27.

Shea, J. J., jun. (1958). Ann. Otol. (St. Louis), 67, 932 (1959). Memphis med. J., 34, 149. (1960). A.M.A. Arch. Otolaryng. 71, 257

Withers, B. T., Richmond, R., and Alford, B. R. (1961). Arch. Otolaryng., 73, 520 .

\section{$\varnothing$ CHRONIC AMOEBIC HEPATITIS \\ BY \\ T. DOXIADES, M.D. \\ Department of Medicine, Evangelismos Hospital, Athens}

\section{[With Special Plate]}

Chronic amoebic hepatitis is the subject of much controversy: many doubt its existence, while others ascribe to it only little clinical significance, and compare it with the post-hepatitic syndrome which occasionally follows viral hepatitis. The predominant conception is that amoebic hepatitis, in general, is a rather rare complication of amoebiasis and constitutes a presuppurative stage.

On the basis of 25 years' experience as a clinician I consider that chronic amoebic hepatitis exists as a distinct entity. It occurs more frequently than is supposed, and is closely connected with the whole problem of amoebiasis.

For the past four years I have collected experimental data and the results of clinical observations which strengthen my concepts on this subject. Since, as most doctors believe (Sherlock, 1958), the pathological basis of these features is not certain, and there are no good reports of hepatic histology, I consider it my duty to describe the results of our observations.

The cases which I shall describe have been taken from material which we have observed and followed in Greece. The reason I emphasize this is because it is known that amoebiasis presents a clinical picture which varies with the geographic location of the countries in which it is found. It will be interesting to determine whether this entity, with or without variations, occurs in other countries. These cases are reported, first, because they are very characteristic, and, secondly, because they will help towards a better understanding of both the clinical features of the condition and the conclusions I have drawn (Doxiades and Candreviotis, 1961).

\section{Case 1}

Four years ago a 32-year-old woman was admitted to the Evangelismos Hospital with generalized oedema and free fluid in the right chest and peritoneal cavity. In the ascitic fluid typical features of Entamoeba histolytica were found on repeated examinations. The ascitic fluid was injected intrahepatically into one guinea-pig and into the portal vein of four others. The guinea-pigs were killed 17 days later. Histological examination of the liver of the animal which had been inoculated intrahepatically revealed an abscess containing an abundance of $E$. histolytica.
Repeated needle biopsies of the patient's liver, also a biopsy specimen of the liver taken at exploratory lapprotomy, showed the presence of $\boldsymbol{E}$. histolytica among the liver cells without any evidence of suppuration. Granulomas containing amoebae were also found.

The patient was given antiamoebic therapy and her condition improved greatly. Several stool examinations for parasites during the above period were negative (Doxiades et al., 1961).

\section{Case 2}

A 48-year-old man was admitted to the hospital on May 14, 1961, having suffered for the past year from loss of appetite, nausea, headaches, fatigue, and weakness accompanied by tenderness and a feeling of fullness in the region of the right hypochondrium. It is worth mentioning that the patient had previously been admitted to another hospital with a diagnosis of carcinoma of the liver.

On clinical examination the liver was found to be enlarged $6 \mathrm{~cm}$. below the right costal margin and firm. Laboratory findings were as follows: Thymol turbidity 3 units; cephalin flocculation $++; \mathrm{ZnSO}_{4}$ turbidity 4 units; serum bilirubin $2 \mathrm{mg}$. per $100 \mathrm{ml}$.; alkaline phosphatase 4.5 units ; " bromsulphalein " retention $8 \%$; serum glutamic oxalacetic transaminase (S.G.O.T.) 36 units; serum glutamic pyruvictransaminase (S.G.P.T.) 60 units. On stool examination $E$. histolyticae were found on two occasions. Proctoscopy did not reveal any pathological lesion. Total protein level and other laboratory findings were within normal limits.

On June 13 needle biopsy of the liver was performed; the specimen presented a histological picture of chronic hepatitis of moderate intensity, characterized by slight enlargement of the portal triads with inflammatory cellular infiltration, mainly by lymphocytes. In some sections small spheroid formations with the morphological features of $E$. histolytica were found, either at the portal triads or in the portal capillaries, among the liver cells (Special Plate, Fig. 1). The liver cells showed moderate degenerative changes and fatty degeneration. No tumour cells were found.

On June 27 an exploratory laparotomy was carried out, in which no tumour was seen. The surface of the liver was strikingly granular, as shown in the accompanying photograph. A small specimen of liver was excised, half of which was examined histologically. This presented the same picture of chronic hepatitis characterized by marked enlargement of the portal areas and showing excessive proliferation of connective tissue, forming bands which divided the liver 